\title{
The challenge of industrializing proteomics
}

\section{To the editor:}

Two special issues of Nature Biotechnology $y^{1}$ and Nature ${ }^{2}$ set out to highlight progress and identify the challenges that lie ahead for industrializing proteomic technologies. In both issues, a rosy picture was painted of the potential of mass spectrometric approaches (e.g., isotope-coded affinity tags; ICAT) to impact efforts to quantitatively investigate molecular physiology. Unfortunately, some of the real obstacles that currently prevent proteomic techniques (including ICAT) from delivering on its promise were understated, particularly as it applies to use in drug discovery. It is important to emphasize this view to manage expectations of such technology, considering that pharma is a top-end consumer and adopter of proteomics.

Quantitative proteomics has much to offer in understanding the physiological consequences played out at the molecular level following inhibition of a drug target. However, the challenges associated with applying quantitative proteomics in this setting are complex. Experiments are multivariate, involving tissues, drugs, time points and physiological models. A typical experiment may investigate two tissues, with two drugs, collecting three time points for five animals (that is, 60 samples). Such an experiment generates mountains of data, which once collected, must be prioritized, filtered, analyzed and assembled. In parallel, business drivers push projects forward, meaning that timelines for delivering results from proteomics (and other technologies) are painfully short and strict.

Overcoming these challenges are real concerns for the industrialization of proteomics. Unfortunately, in their current incarnation, quantitative mass spectrometric approaches are far too slow to be considered practical, efficient solutions for operating in such an environment. The problem lies not with the approach, rather, in transitioning the data into knowledge. As pointed out by Patterson" ${ }^{3}$, "data analysis is the Achilles heel of proteomics" and "advances will require intensive efforts on the computational front."

These are solvable problems that need attention to move this approach into the mainstream. In the meantime, for all their drawbacks, two-dimensional gels remain a reliable, although undesirable option, to address throughput for quantitative proteomics. Identifying proteins and measuring their expression are only the initial steps along a far lengthier path in using the outputs from proteomics for decision-making in drug discovery. Ascribing functional attributes to proteins of interest is key, and assembling the reams of data produced in a proteomics experiment (and complementary parallel experiments) into pathways of physiology is essential to generate testable hypotheses. In combating these challenges we are only just testing the waters. Our knowledge of protein function is frustratingly weak, and it is only through tenacious, laborious efforts that we can expect to expand it. Solutions to these challenges need to be at the forefront of efforts to industrialize proteomics.

\section{Mark P. Molloy}

Pfizer Global Research \& Development, Ann Arbor Laboratories, Ann Arbor, MI 48105, USA e-mail:mark.molloy@pfizer.com

\footnotetext{
1. Challenges in proteomics. Nat. Biotechnol. 21, 213-340 (2003).

2. Insight: Proteomics. Nature 422, 191-237 (2003).

3. Patterson, S.D. Nat. Biotechnol. 21, 221-222 (2003).
}

\section{Clarifying press before paper}

\section{To the editor,}

Although we agree with the overall goal of Neal Stewart's recent Commentary entitled 'Press before paper-when science and media collide' (Nat. Biotechnol. 21, 353, 2003), we object to several of his statements. In particular, we strongly disagree with his view that an Ohio State University press release describing our work on wild sunflower could have contributed to unnecessary deaths by starvation in Africa. Stewart's analysis of the food aid crisis in Africa is ludicrous. This situation is far too long-standing and complex to be swayed by a paper describing the potential effects of trangenes in wild populations. In addition, his association of our work with claims about cloned human babies is insulting.

The goal of Stewart's commentary was to point out the dangers of discussing scientific results with the media before they have been published in the peer-reviewed literature, and he criticized the fact that our presentation at a 2002 scientific meeting was featured in a university press release

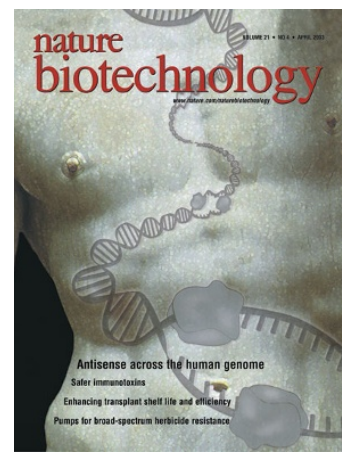
before it was published. We would have preferred to have had a publication coincide with the meeting, but the novelty of our findings and the year-or-longer delays of most peer-reviewed journals prompted us to agree to a carefully crafted press release (http://www.osu.edu/research news/archive/sungene.htm). Stewart's inflammatory commentary gives the impression that our actions were irresponsible at best or some kind of hoax at worst. This is disingenuous because our paper was accepted for publication months ago and has just been published ${ }^{1}$. In fact, Stewart attended a workshop at which we described these experiments in 